Voix et Images

volxetimages

\title{
Carrefours du féminisme : pour un éloge de l'errance
}

\section{Catherine Mavrikakis}

Volume 30, numéro 1 (88), automne 2004

Le pseudonyme au Québec

URI : https://id.erudit.org/iderudit/009896ar

DOI : https://doi.org/10.7202/009896ar

Aller au sommaire du numéro

Éditeur(s)

Université du Québec à Montréal

ISSN

0318-9201 (imprimé)

1705-933X (numérique)

Découvrir la revue

Citer ce compte rendu

Mavrikakis, C. (2004). Compte rendu de [Carrefours du féminisme : pour un éloge de l'errance]. Voix et Images, 30(1), 139-147.

https://doi.org/10.7202/009896ar d'utilisation que vous pouvez consulter en ligne.

https://apropos.erudit.org/fr/usagers/politique-dutilisation/ 


\section{F É M I N I S M E S}

Carrefours du féminisme: pour un éloge de l'errance $++$

\section{CATHERINE MAVRIKAKIS}

Université de Montréal

Quel espace théorique se dessine aujourd'hui? Quel territoire imaginaire habitonsnous en tant que critiques? Dans quelle enclave tenons-nous la pensée? Quelle route prendre pour parcourir une question? Et doit-on faire le tour d'une problématique comme on fait, en propriétaire, le tour de son jardin? Ce sont ces questions de l'espace comme épistémologie de la critique que je me suis posées à la lecture du livre collectif Sexuation, espace, écriture. La littérature québécoise en transformation ${ }^{1}$. Sous la direction de Louise Dupré, Jaap Lintvelt et Janet M. Paterson, ce recueil de textes se veut le lieu d'une ouverture de l'aire critique de la sexuation sur de nouvelles perspectives concernant la question de l'espace. Si le mot sexuation est déjà lui-même porteur d'un autre territoire et d'un découpage de la pensée du genre et s'il veut inscrire dans le domaine francophone, en se l'appropriant et en le transformant, le lieu devenu commun et international des gender studies, articuler l'espace à ce concept de sexuation à l'intérieur d'une exploration théorique de la littérature se donne comme novateur et cherche à circonscrire d'inexplorés secteurs des études québécoises.

Issue d'un colloque sur l'espace et la sexuation dans la littérature québécoise, qui eut lieu à l'Université de Groningue aux Pays-Bas, en mai 2001, cette collection de textes est conçue à la fois comme un haut lieu de la réflexion sur le genre au Québec et comme une terre inconnue aux contours et frontières encore mal définis dont les études ultérieures viendront préciser ou défaire les limites. Il y a dans le geste de ce livre et du colloque qui le précédait l'idée de la fondation d'un nouveau champ disciplinaire que l'on veut fertile en lectures et relectures. Il y a dans l'acte d'élaboration de cet ouvrage une véritable tentative de territorialisation de la critique qui se donne pour but, comme c'est si souvent le cas, d'aborder autrement les problèmes, ce qui apparaît manifeste dans la rhétorique de la nouveauté qui parcourt l'introduction. Il s'agit aussi de suivre les mouvements de la littérature

$$
++
$$

1 Louise Dupré, Jaap Lintvelt et Janet M. Paterson (dir.), Sexuation, espace, écriture. La littérature québécoise en transformation, Québec, Nota bene, 2002, 484 p. 
québécoise. Celle-ci est décrite comme «dynamique et audacieuse» et surtout capable «de suivre l'évolution de la pensée contemporaine et de créer de nouveaux espaces réels et symboliques marqués par la sexuation" (21). Or, à lire le livre, on s'apercevra qu'il ne s'agit pas tant d'analyses de textes actuels que de lectures renouvelées de textes plus largement contemporains, écrits par des auteurs souvent bien établis (par exemple Yves Thériault, Anne Hébert ou encore Yolande Villemaire). $C^{\prime}$ est l'image de l'auberge espagnole qui vient ici à l'esprit quand il s'agit de comprendre la place réservée au lecteur dans ce recueil. Celui-ci, en effet, n'y trouvera rien d'autre que ce qu'il a voulu y apporter ou y chercher. En ce sens, aucune somme totalisante, aucune réflexion d'ensemble ne lui sera proposée et l'introduction insiste précisément sur cette multiplicité des points de vue, sur la construction d'un espace critique hétérogène où les textes se répondent parfois, se font échos les uns des autres, et jouent à cache-cache théoriquement entre eux.

Louise Dupré dans son texte intitulé «Entre raison et déraison de France Théoret. Esquisse d'une cartographie de l'écriture» parle d'espace de circulation, de territoire psychique de l'entre-deux et de découpage de l'espace littéraire à inventer. Ce que Dupré décrit ici avec beaucoup de justesse à propos des écrits de Théoret pourrait servir de matrice intellectuelle à la compréhension de tout l'ouvrage présenté. Lieu de passage, Sexuation, espace, écriture crée des lieux de l'entre-deux qui parviennent à généraliser l'instabilité de toutes les énonciations en multipliant celles-ci. L'espace imaginaire de la théorie de ce recueil ressemble alors à un corridor, un couloir, une antichambre conceptuelle où le lecteur attendrait en vain une épistémologie de la question ou une vue d'ensemble. Il s'agit plutôt d'un espace-livre où les courants d'air peuvent être nombreux, mais où l'on peut respirer, humer de nouvelles odeurs, errer, déambuler et se promener au gré de son désir et de ses intérêts. Le livre forme un dispositif spatial où le mouvement est souverain et dans lequel on entre et sort à sa guise. Les vingt-deux différents articles qui le constituent sont plus centrés sur un auteur et une problématique que sur une question plus grande concernant la sexuation vue dans son étendue diachronique et synchronique. Inscrire la particularité, la spécificité d'une ou de quelques écritures reste souvent le principal but de ces parcours critiques. Des hommes et des femmes travaillent ici sur des auteurs féminins et masculins et ne cherchent aucunement à s'arroger le dernier mot sur la littérature québécoise, pas plus qu'à éviter le mauvais genre d'une position théorique. Des voix se font ici entendre sans qu'aucune ne tente de s'approprier un espace de la parole fortement idéologique. Si un sous-texte féministe est à lire à travers cet ouvrage, si le recueil se pose d'emblée dans la mouvance d'un néo-féminisme, force est de constater que le ton de ces études est très scientifique et se tient loin de toute polémique ou prise de position violemment identitaire. La provenance des auteurs des différents chapitres participe de cette volonté d'aménager un territoire ouvert de la pensée. Du Québec, des États-Unis, du Canada, des Pays-Bas, les chercheurs venus d'espaces éloignés les uns des autres sont rassemblés pour couvrir un territoire critique aux origines théoriques diverses. Le fait que le colloque qui a présidé à l'idée de ce livre ait eu lieu hors du Québec participe peutêtre de cette nécessité de déplacement de la pensée et de nomadisme de la 
réflexion qui a besoin de se décentrer pour faire advenir de nouvelles positions intellectuelles. Il y aurait là un travail certain de l'altêrité.

La domestication, l'appropriation de l'espace ou son caractère fondamentalement inaliénable, reste un des enjeux majeurs des études ici développées. C'est le travail du politique qui s'instaure dans la prise de possession de la ville, du monde et du temps. Mary Jean Green dans «Cartographies de la mémoire, réinscription du féminin : l'autofiction chez Régine Robin » dégage l'idée de la flâneuse. Cette dernière, dans la lignée baudelairienne, au cours de ses pérégrinations, dessine les contours montréalais d'une postmodernité où la femme se retrouve au cœur d'un processus d'investigation de l'espace. Jaap Lintvelt, lui, s'attaque à une vingtaine de romans où la ville de Québec est vécue par des personnages masculins et féminins pour lesquels les lieux urbains sont investis différemment de connotations d'enfermement ou de protection. L'écriture apporte alors une libération sociale qui n'est pas sans lien avec sa sexuation, comme Lintvelt nous le démontre dans «Expériences féminines et masculines de l'espace dans la ville de Québec». La ville est conçue comme lieu de répression ou encore de développement social. «Dans l'espace rebelle et le sexe faible: figures de la femme patriote de la rébellion 1837-1838", Marilyn Randall nous permet, par une approche plus historique, de comprendre qu'il existait, dans la tradition agricole populaire, l'idée d'un espace commun aux hommes et aux femmes: l'espace domestique où s'abolissaient les frontières entre le privé et le public. La place centrale de la femme dans cet espace explique l'idée fausse de l'absence féminine dans la rébellion de 1837-1838. L'histoire aurait négligé toute une sphère d'activités exclues des domaines publics et a, en quelque sorte, rendu invisible la participation des femmes dans l'histoire en effaçant le lieu familier de toute révolution: la maison.

Avec son très bel article sur un Nord inventé, François Paré montre quelles fictions fondatrices mettent en scène des amorces et des absences de solutions identitaires. Le nord de l'Ontario a été bien sûr le lieu réel de l'implantation de migrants québécois à la fin du XIX siècle et au début du XXe siècle. Mais la constitution imaginaire dans une culture franco-ontarienne d'un espace nordique collectif et rassembleur se calque totalement sur un discours littéraire national québécois qui a un rapport naturel et politique au pays du froid et de la neige. Le Nord comme espace mélancolique de l'origine perdue, comme lieu du chant permet une indifférenciation de l'identité et échapperait à «la binarité épuisante de l'espace» (272).

Ce sont la filiation et l'Histoire qu'elle déploie qui se trouvent analysées à travers maints articles, dans une conjugaison très particulière du lieu et du temps. Dans «Les espaces impossibles de la relation père-fille», Lori Saint-Martin montre combien la place du père dans la littérature québécoise est problématique et impensée. Cette place serait non seulement symbolique mais aussi elle serait liée aux lieux que le père peut ou ne peut pas investir. C'est la figure paternelle dans une certaine domesticité et un enfermement qui travaille le roman de Gaétan Soucy, La petite fille qui aimait trop les allumettes, qu'analyse Janet M. Paterson. Quand, à la fin du roman, l'écriture passe entièrement au féminin, il est possible de dire que dans l'espace du récit apparaît l'existence d'un nouveau sujet du discours, pour lequel l'énonciation est fondamentale. Ce sujet interroge les concepts d'identité et 
d'altérité par l'intermédiaire d'une interrogation perpétuelle sur des dispositifs spatiaux. Dans «Espace autofictif, sexuation et deuil chez Denise Desautels et Paul Chanel Malenfant», Barbara Havercroft se penche elle aussi sur la question d'un territoire généalogique où peut s'élaborer l'héritage symbolique laissé par nos parents, nos proches. Sur la performativité de l'écriture, son agentivité, Havercroft s'attardera et pensera l'écriture comme lieu où le sujet blessé, endeuillé est à même de se réinventer, de réparer les trous de sa vie et d'agir sur le monde. Contrairement à ce qui se passe dans l'article de Saint-Martin, les textes de Paterson et de Havercroft voient l'écriture non seulement comme un symptôme d'un malaise culturel ou généalogique, mais aussi comme un espace de guérison, comme une enclave où le pacte social peut être transformé ou renégocié. C'est ce que propose aussi Robert Schwartzwald dans ses «Cartographies de la sexuation dans The Dragonfly of Chicoutimi ». Analysant le monologue théâtral de Larry Tremblay, Schwartzwald montre la contamination par l'anglais du récit de Gaston Talbot qui ne peut plus dire le mot «Cheval» mais bien plutôt prononce «Horse» en soliloquant dans un anglais boiteux. La place du public qui assiste à une pièce donnée en français dans un thêâtre français alors qu'on y parle anglais devient problématique pour Schwartzwald. Celui-ci montre ainsi combien l'espace thêâtral ici compromet le spectateur et brouille les repères identitaires durant la représentation. Le texte est alors un acte, un travail sur la perte possible de l'identité.

Sexuation, espace, écriture aménage un monde habitable pour la pensée sur le genre et le gender. Ce lieu qui accueille diverses paroles et de nombreuses questions ne peut être conçu comme un espace où l'on assisterait simplement à la domestication, la familiarisation de la théorie. L'agencement d'un nouveau territoire critique demande à ceux qui s'y attachent de quitter la place aisée de pionniers et de pionnières du champ. Il faut penser l'espace comme un lieu de pérégrinations, d'errances et de voyages insensés. À travers une absence certaine de discours unificateur ou univoque, les directeurs de cet ouvrage, Louise Dupré, Jaap Lintvelt et Janet M. Paterson, ont pris le parti de vouloir à la fois créer un nouveau territoire d'élaboration de la différence sexuelle et construire un espace troué, un espace déterritorialisé qui veut s'éloigner de toute esthétique classique du paysage familier. Dans ce monde ainsi dessiné, on peut se perdre peut-être, mais aussi faire de précieuses rencontres. Les chemins qui le sillonnent peuvent sembler ne mener nulle part ou encore conduisent vers un espace mal défini, mais il est impossible de ne pas les suivre, de ne pas s'y aventurer.

C'est cette même possibilité d'errance que le lecteur doit affronter dans le recueil de textes assemblés par Isabelle Boisclair, Lectures du genre ${ }^{2}$. Le pluriel du titre montre assez que les voies ouvertes pour l'interprétation du texte et du genre qui le tra-

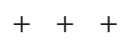

2 Isabelle Boisclair (dir.), Lectures du genre, Montréal, Les éditions du remue-ménage, 2002, 179 p. 
vaille ou encore du genre qu'il travaille seront multiples et même parfois divergentes. Néanmoins, il s'agit de prêter attention aux différentes manifestations du genre dans le texte et de remettre en question encore et toujours le binarisme de la différence sexuelle. Femmes littéraires, encore un effort avant d'être féministes! C'est un peu ce qui semble être le mot d'ordre de cet assemblage de réflexions qui veulent démonter la construction des dispositifs philosophiques, littéraires et sociaux dans la pensée des sexes. Or, et Boisclair insistera avec raison là-dessus, il ne s'agit pas seulement de mettre en évidence, d'accompagner une conscience du genre mais aussi de faire réfléchir sur «l'inconscient de genre» qui fait parler les textes sans qu'ils sachent toujours ce qu'ils disent. Si l'indicible féminin travaille plusieurs contributions de ce livre, force est de constater que l'indicible côtoie le non-dit, le refoulement, la dénégation et tout travail incessant de l'idéologie dans les mots. Traquer cette prégnance de la division sexuelle, tel serait le but de ce livre afin de faire se déployer le sens dans sa multiplicité, sa complexité et dans ses vagabondages.

Le texte de Louise L. Larivière en ouverture du recueil permet de réfléchir sur le processus de féminisation linguistique qui a lieu au Québec depuis les années quatre-vingt. Sans être dogmatique, Larivière propose un cadre à la féminisation en comparant la façon de procéder ici au regard des usages dans d'autres pays francophones. Or, Larivière conclut à la nécessité d'une normalisation mondiale du genre. Il s'agit de mettre de l'avant que la diversité des pratiques en matière de féminisation porte atteinte à l'apprentissage du français. Même si cette prise de position de la linguiste qu'est Larivière va dans le sens d'une pensée de la langue comme lieu politique, comme appropriation d'un territoire social à conquérir, on peut s'étonner à juste titre de voir que, dans un livre intitulé Lectures du genre, apparaisse un discours sur l'uniformisation du féminin dans la langue. On est alors loin d'une volonté de dépasser le binarisme, puisqu'au contraire, on veut asseoir celui-ci dans un code qui fonctionnerait de façon égalitaire et efficacement double. On se demande alors si la déconstruction ou l'anti-binarisme théorique à l'œuvre dans un tel recueil ne sont pas des pétitions de principe pour littéraires et doivent trouver leurs limites dans des disciplines où la réalité et le contexte sont plus importants. Sans vouloir critiquer d'aucune façon le texte de Larivière, je crois que sa présence dans ce livre montre combien la littérature est parfois, et malgré ce qu'elle peut en dire, en porte-à-faux, presque sans lien avec des discours basés sur des statistiques et des démonstrations comptables. La place inaugurale du texte de Larivière, "Quand une langue se donne un genre ou de la bonne féminisation linguistique», laisse penser que la multiplicité du genre n'est possible que dans cette fondation d'une langue stable, d'une «bonne» féminisation au sein de laquelle le binaire serait précisément advenu. De même, Sylvie Pelletier, dans son texte sur l'autobiographie d'Honorine Tyssandier (1828-1894), cherche à voir comment s'exprime la conscience de genre, la conscience d'être une femme pour une Française de la seconde moitié du XIX siècle. Il n'est donc pas question de dépasser un rapport duel aux hommes, un binarisme du genre, mais au contraire de faire naître à l'intérieur de cette structure binaire l'in-su, le refoulé, le méprisé que constitue la féminité. On peut dire que la sortie de la binarité n'aura vraiment lieu 
qu'avec les lectures de textes beaucoup plus récents et que, là, l'errance du sens, sa multiplicité grâce à laquelle on se perd sans cesse, puis on se retrouve, a lieu. Évelyne Ledoux-Beaugrand, Martine Delvaux, Isabelle Boisclair dans leurs approches plurielles d'Annie Ernaux, de Camille Laurens et de Nancy Huston nous conduisent davantage, à tâtons, dans une baguenaude maîtrisée, vers une nouvelle métaphysique où il y aurait une véritable mise en cause des postulats phallocentriques. La difficulté à s'orienter dans la pensée en marche a quelque chose d'absolument séduisant où le lecteur fait confiance à la main qui le tient durant cette promenade périlleuse. C'est cette question de la subversion, des voies non empruntées par la littérature qu'examine Sylvie Mongeon dans son étude d'Amadou (1963) de Louise Maheux-Forcier. L'effraction du symbolique par le sémiotique permet de passer de la signification au processus même de la signifiance et de «dé-nommer» le monde au lieu de le nommer, de le baptiser pour le posséder. Il faut alors se perdre au sein même de la langue. La dissidence est au cœur du travail de la romancière Maheux-Forcier et a marginalisé celle-ci dans un Québec qui n'avait d'autre désir à l'époque que celui de combler ses failles et de fonder une identité monolithique qui se lit aisément à même les romans écrits par des hommes des années soixante. La langue comme espace de dés-appropriation du masculin intéresse aussi Sandrina Joseph dans son texte sur Nécessairement putain de France Théoret. En dotant la langue de la narratrice de mots injurieux, d'obscénités, Théoret fait franchir un seuil aux femmes. La prostituée, la putain prend la parole qui la blesse, la domine et s'en pare. Elle reproduit le code masculin pour le couper de son origine et le subvertir. Elle emprunte les chemins de traverse et les travers masculins afin de faire du langage un lieu ouvert à tous. L'érotisme au féminin, dans les romans des années quatre-vingt, est étudié par Élise Salaün. Si celle-ci nous parle de l'émergence du corps masculin dans les livres de femmes, elle insiste aussi sur le lien entre la jouissance physique et le plaisir de l'écriture qui donne naissance au sacré et à un mysticisme incarné. Enfin, Lucie Joubert trace un portrait de Stéphane Venne, "L'homme qui faisait chanter les femmes». Venne a en effet signé paroles et musiques pour maintes femmes artistes. À travers la lecture des chansons d'Isabelle Pierre, de Renée Claude et d'Emmanuelle, Joubert nous montre comment Venne a contribué aux mutations sociales en permettant à beaucoup de filles de fredonner, mine de rien, le début de leur liberté. Le refrain féministe, on l'aura compris, est nécessaire. Et je pourrai dire que Lectures du genre est une espèce de ritournelle d'interprétations, de rengaines qui nous reviennent en mémoire, qui nous viennent à l'esprit sans que l'on s'en aperçoive dès que l'on lit les textes à l'épreuve de la différence sexuelle. Ce recueil a un air de chanson bien connu puisqu'il viendrait nous donner des nouvelles des lectures du féminisme à l'heure actuelle, en répétant, il va sans dire, pas mal de lieux communs que l'on doit sans cesse réhabiter, déplacer, défaire. Or, c'est dans ces redites, dans cette façon de s'attaquer encore et toujours à des problèmes importants que ce livre tire son originalité. Il n'a pas la prétention de tout fonder, de tout inventer, mais plutôt de produire une pensée de la faille, une pensée qui permettra de penser autrement. Le piétinement, la reprise, la ritournelle sont au cœur d'un projet de déplacement de la différence sexuelle. D'un texte à l'autre, des échos se font entendre, des voix se 
mêlent, se confondent ou se font concurrence afin de parvenir à montrer quelque chose de la cacophonie des voix sexuées, du féminin comme espace discordant, comme possibilité de sa propre errance.

Dans son livre sur «le rire des filles», sous-titre qu' elle donne à son ouvrage L'humour du sexe ${ }^{3}$, Lucie Joubert nous amuse, nous fait sourire et parfois nous gondoler... Il faut avouer que le sujet s'y prête, non pas tant parce qu'il y a là matière à imitation, adaptation au thème abordé, contagion hystérique ou démonstration, mais bien plutôt parce que la faible présence des femmes dans le domaine de l'humour a de quoi faire rigoler et même rire jaune! Le parti pris de Joubert est clair: il s'agit de comprendre pourquoi on répète à satiété que les femmes ne savent pas s'amuser et de prouver fortement le contraire en mettant en relief une histoire des femmes humoristes au Québec dans différents groupes et revues. Le rire médusant des femmes, celui que l'on fantasme comme castrateur ou tout simplement coupant, a été en quelque sorte endigué par un onzième commandement, celui-là tout spécialement destiné à la gent féminine: "Tu ne riras point.» Tabou des excrétions et sécrétions, des excès d'une féminité qui déborderait de partout, dont le corps abandonné au rire et au fou rire dérange, agresse. Dans l'humour en groupe au féminin, gît la possibilité d'une conspiration entre femmes, une manifestation carnavalesque du monde, une levée des inhibitions que Joubert voit à l'œuvre dans l'humour de cuisine où la bouffonnerie est de rigueur et où le banquet à venir, le vin siroté délient les langues et libèrent les esprits. Si le banquet a toujours été une réunion masculine, les ripailles des femmes sont l'occasion d'une subversion certaine de l'ordre, mais gardent encore un côté privé, puisqu'elles n'ont pas atteint la notoriété du Banquet de Platon ou de la partie de chasse consacrée aux liens virils. Les Folles Alliées, troupe de théâtre à vocation féministe, a parcouru le Québec de 1980 à 1990 pour faire entendre précisément des créations collectives, un humour conçu en communauté, pour faire communauté. Il s'agissait de créer ici et là, un soir, le temps d'un spectacle, une société de femmes où le rire était fondateur. Au moment où les grands rassemblements sont choses du passé, des femmes forment de petits groupes pour rire, pour penser, pour dénoncer en tentant de faire apparaître de nouvelles expressions du féminisme. De même, les responsables de revues, comme La Gazette des femmes ou La Vie en rose, ont tenu à faire entendre dans des chroniques humoristiques une voix individuelle qui parle du féminisme non seulement comme projet ou idéologie, mais aussi comme singularité, comme déception ou espoir. Il s'agissait de rendre les revues moins didactiques en octroyant à une parole plus officieuse la possibilité de servir les enjeux féministes tout en laissant place à du doute, du jeu, du rire. Suzanne Jacob, qui s'occupa donc de la chronique $A h ! .$. pendant près de dix ans, de 1981 à 1991, a permis à bon nombre

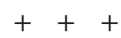

3 Lucie Joubert, L'humour du sexe. Le rire des filles, Montréal, Triptyque, 2002, 191 p. 
de lectrices de pouvoir se moquer d'elles-mêmes, des féministes et de se trouver désarçonnées dans leur conviction politique intime. La pratique d'une voix féminine pleine de paradoxes pourrait être bien sûr pensée comme un symptôme du backlash qui frappa dans les années quatre-vingt. Mais elle permet à la doxa de s'incarner dans une figure qui parfois est elle-même aux prises avec la réalité du quotidien. Un peu différente est, pour Joubert, Hélène Pednault dans ses Chroniques délinquantes de La Vie en rose ou encore à la radio. Pednault a su faire rire dans ses attaques incessantes contre les ennemis du féminisme et a eu ces remarques pour le moins saisissantes:

Il arrivera, entre ces pages, que certaines chroniques n'auront pas été écrites pour faire rire. Si vous riez quand même, je ne peux rien faire pour vous: soit votre rire est devenu un réflexe incontrôlable, à l'instar du hoquet, soit vous êtes atteint du réflexe conditionné Juste pour rire, le Pavlov québécois. Incurable 4 .

À travers cet essai sur le rire au féminin, Joubert elle-même, s'inspirant assurément de Pednault, nous invite à saisir ce qu'il en est de notre propre rire forcé, discret ou heureux, d'en voir les mécanismes et de nous familiariser davantage à un rire pratiqué par les femmes qui parfois peut rendre mal à l'aise certaines d'entre elles, conditionnées comme elles le sont à une pléthore de blagues sexistes (les meilleures, n'est-ce pas?) ou à divers stéréotypes désopilants. Joubert pense que le rire, cela s'apprend, cela se développe et que les filles, de par leur éducation vertueuse et pudique, n'y ont pas eu droit. Quand elles ont eu la possibilité de s'esclaffer un peu, elles ont dû rire des blagues qui se faisaient à leurs dépens et ne peuvent que depuis très peu de temps s'éduquer à un autre type d'humour, fréquenter d'autres écoles du rire et rigoler de bon cœur des travers des hommes et même, pourquoi pas, faire dans l'autodérision et la critique intelligente de soi. Les femmes savent rire. C'est bon à savoir...

Cet essai donne à lire de nombreux extraits humoristiques qui prouvent de façon incontestable que les filles se dilatent la rate, se fendent la pipe, se tordent les côtés et même parviennent à faire rire. Édifiant, non? Or, c'est le lieu de notre propre humour, des limites de celui-ci, de notre hardiesse, de notre capacité à la provocation que Lucie Joubert veut interroger à travers le florilège de citations et d'anecdotes qu'elle offre aussi dans le livre. Elle-même se raconte. À Paris, à la présentation d'un spectacle d'Anne Roumanoff qui demandait à la salle de lui lancer des insultes et des injures afin de s'endurcir dans son futur métier de prof, Joubert a été quelque peu secouée par sa voisine qui a lancé tout de go à l'humoriste un «va te faire enculer» retentissant. Roumanoff a alors pris Joubert à témoin en lui disant: «Ouais, on commence fort ce soir!» Joubert analyse ici sa gêne, sa difficulté à se laisser aller au comique de la situation et ses propres réflexes de rigolote un peu

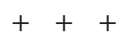

4 Hélène Pednault, Chroniques délinquantes de La Vie en rose, Montréal, VLB éditeur, 1988, p. 9. Cité par Lucie Joubert, p. 137. 
coincée, malgré une pratique effrénée du rire théorique et pratique. De même, elle propose un exercice qui permettra au lecteur d'évaluer «sa propre réaction» devant certains types d'humour féministe qui vont des blagues légères aux plus acerbes et engagées. En fait, l'essai de Joubert veut parvenir à analyser les mécanismes contemporains d'un rire sexué et faire advenir une parole féminine et féministe amusante qui sait explorer toutes les nuances du bidonnage: la farce grasse et vulgaire tout aussi bien que le rire savouré à petite dose de sagacité. Néanmoins, Joubert veut nous prévenir contre les pionnières de l'humour qui calqueraient exclusivement leur travail sur les blagues de gars et qui se contenteraient de «reproduire en mode inversé le discours masculin en s'amusant des beaux-pères, de l'obsession des voitures des hommes, etc.» (34). Cet essai veut faire apparaître un nouveau territoire, décolonialiser le rire et lui permettre de contester un certain ordre établi au lieu de l'encourager même à s'institutionnaliser sans cesse dans la joie bébête et sexiste. Je pense ici à ce que Joubert dénonce comme l'autodérision personnelle, une espèce de self-bashing qui fait toujours sourire, mais qui a néanmoins des ancrages sociopolitiques assez dangereux. Cette pudeur féminine, cette tenue au secret, à l'humilité, voire à l'humiliation, qui caractérisent la position des femmes changent avec les avancées du féminisme. L'histoire de l'humour des femmes est alors absolument connexe aux progrès sociaux et reste un indice d'une réflexion à mener sur la gravité, parfois contrainte, des mouvements révolutionnaires et sur la capacité de ceux-ci à se fonder autrement que par des déclarations de principe. Le changement souhaité, rêvé, passe par les objets de prédilection du rire et du sérieux dans le social. Il s'inscrit dans notre façon de nous amuser, dans notre capacité à faire voler en éclats de rire un discours politique compassé ou même trop facilement hilarant. 